\title{
Energy states of colored particle in a chromomagnetic field
}

Sh. Mamedov ${ }^{\mathrm{a}}$

Institute for Physical Problems, Baku State University, Z. Khalilov str. 23, 1148, Baku, Azerbaijan

Received: 12 August 2007 /

Published online: 28 September 2007 - (C) Springer-Verlag / Società Italiana di Fisica 2007

\section{Erratum to:}

Eur. Phys. J. C 49, 983-995 (2007)

DOI 10.1140/epjc/s10052- 006-0173-7

We aim to correct sign misprints in Sect. 3 of this paper.

1. “干” in the expressions (35) and above for the energy spectra $E_{1,2}^{2}$ should be read as " \pm ".

2. Signs before the last addendants in the diagonal elements $h_{11}^{\prime}$ and $h_{55}^{\prime}$ in the equation (39) should be changed and the corrected equation (39) has the following form:

$$
h_{11}^{\prime} \equiv f_{3}=\mathcal{P}^{2}+\mathcal{G}^{2} / 4+\mathcal{G} p,
$$

$$
\begin{aligned}
h_{22}^{\prime} \equiv f_{6} & =\mathcal{P}^{2}+5 \mathcal{G}^{2} / 4-\mathcal{G} p^{\prime}, \\
h_{33}^{\prime} \equiv f_{1} & =\mathcal{P}^{2}, \\
h_{44}^{\prime} \equiv f_{5} & =\mathcal{P}^{2}+5 \mathcal{G}^{2} / 4+\mathcal{G} p^{\prime}, \\
h_{55}^{\prime} \equiv f_{4} & =\mathcal{P}^{2}+\mathcal{G}^{2} / 4-\mathcal{G} p, \\
h_{66}^{\prime} \equiv f_{2} & =\mathcal{P}^{2} .
\end{aligned}
$$

These misprints do not lead to any change and modifications in the other expressions and calculations of this paper. 